\title{
Permanence and Extinction of a Stochastic Delay Logistic Model with Jumps
}

\author{
Chun Lu ${ }^{1,2}$ and Xiaohua Ding \\ ${ }^{1}$ Department of Mathematics, Harbin Institute of Technology (Weihai), Weihai 264209, China \\ ${ }^{2}$ School of Science, Qingdao Technological University, Qingdao 266520, China \\ Correspondence should be addressed to Xiaohua Ding; whmathdxh@163.com
}

Received 3 October 2013; Revised 10 December 2013; Accepted 25 December 2013; Published 16 February 2014

Academic Editor: Asier Ibeas

Copyright (c) 2014 C. Lu and X. Ding. This is an open access article distributed under the Creative Commons Attribution License, which permits unrestricted use, distribution, and reproduction in any medium, provided the original work is properly cited.

\begin{abstract}
This paper is concerned with a stochastic delay logistic model with jumps. Sufficient and necessary conditions for extinction are obtained as well as stochastic permanence. Numerical simulations are introduced to support the theoretical analysis results. The results show that the jump process can affect the properties of the population model significantly, which conforms to biological significance.
\end{abstract}

\section{Introduction}

Recently, Freedman and Wu [1] considered the following delay logistic model:

$$
\frac{d x(t)}{d t}=x(t)[r(t)-a(t) x(t)+b(t) x(t-\tau(t))]
$$

where $r(t)$ is the net birth rate, $a(t)$ denotes the self-inhibition rate, $b(t)$ represents the reproduction rate, and $\tau(t)$ is the time-varying delay. There is an extensive literature concerned with the properties of system (1) and we here mention [2-4] among many others.

As we know, stochastic population models have recently been investigated by many authors (see, e.g., [5-10]). Particularly, May [5] has revealed that due to environmental noises, the growth rate should be stochastic. Suppose that the growth rate $r(t)$ is perturbed by white noise (see, e.g., $[9,10]) r(t) \rightarrow$ $r(t)+\sigma(t) x^{\theta}(t) \dot{\omega}(t)$ where $\dot{\omega}(t)$ is the white noise, namely, $w(t)$ is a Brownian motion defined on a complete probability space $(\Omega, \mathscr{F}, \mathscr{P})$ with a filtration $\left\{\mathscr{F}_{t}\right\}_{t \in \bar{R}_{+}}$satisfying the usual conditions; $\sigma^{2}(t)$ represents the intensity of the white noise. As a result, (1) becomes the following model:

$$
\begin{aligned}
d x(t)= & x(t)[r(t)-a(t) x(t)+b(t) x(t-\tau(t))] d t \\
& +\sigma(t) x^{1+\theta}(t) d w(t) .
\end{aligned}
$$

On the other hand, the population may suffer from sudden environmental shocks, for example, massive diseases like avian influenza and SARS, earthquakes, hurricanes, epidemics, and so forth. Bao et al. [11, 12] and Liu and Wang $[13,14]$ incorporate a jump process into the underlying population system, which can describe these phenomena well and provide a more practical model. Particularly, the books by Applebaum [15] and Situ [16] are also good references in this area. Furthermore, some scholars have researched the theory about stochastic differential delay equations with jumps recently (see, e.g., [17-19]). However, as far as our knowledge is concerned, no articles on introducing a jump process into stochastic delay population has been introduced. Motivated by these, we will consider the stochastic delay logistic model with jumps:

$$
\begin{aligned}
d x(t)= & x(t)[r(t)-a(t) x(t)+b(t) x(t-\tau)] d t \\
& +\sigma(t) x^{1+\theta}(t) d w(t) \\
& +x\left(t^{-}\right) \int_{\mathbb{V}} \gamma(u) \widetilde{N}(d t, d u),
\end{aligned}
$$

with the initial data $\xi(t) \in C_{\mathscr{F}_{0}^{b}}\left([-\tau, 0] ; R_{+}\right)$, where $C_{\mathscr{F}_{0}^{b}}\left([-\tau, 0] ; R_{+}\right)$denotes the family of all bounded, $\mathscr{F}_{0}$-measurable, $C\left([-\tau, 0] ; R_{+}\right)$-valued random variables and $R_{+}=$ $(0,+\infty)$. Here, $x\left(t^{-}\right)=\lim _{s \uparrow t} x(s), N(d t, d u)$ is a real-valued 
Poisson counting measure with characteristic measure $\lambda$ on a measurable subset $\mathbb{Y}$ of $\bar{R}_{+}$with $\lambda(\mathbb{Y})<+\infty, \widetilde{N}(d t, d u)=$ $N(d t, d u)-\lambda(d u) d t, \gamma(u)$ is bounded function, and $\gamma(u)>$ $-1, u \in \mathbb{Y}$. Furthermore, we assume that $w(t)$ is independent of $N$ and $\tau(t) \equiv \tau$ is nonnegative constant.

Based on the fact that model (3) describes a population dynamics, it is very important to investigate the permanence and extinction. The main aims of this work are to investigate how jump process affect the permanence and extinction of model (3). Our results demonstrate that the jump process can change the permanence and extinction, which accords with biological significance. In addition, we establish the sufficient and necessary conditions for stochastic permanence and extinction of model (3).

For model (3) we always assume the following.

(A1) $\theta>0.75, r(t), a(t), b(t)$, and $\sigma(t)$ are continuous bounded functions on $\bar{R}_{+}$with $\inf _{t \in \bar{R}_{+}} a(t)>0$, $\inf _{t \in \bar{R}_{+}} b(t)>0$, and inf $\operatorname{i\in }_{t} \sigma(t)>0$, where $\bar{R}_{+}=$ $[0, \infty)$.

(A2) For each $m>0$ there exists $L_{m}$ such that $\int_{\mathbb{Y}} \mid H(x, u)-$ $\left.H(y, u)\right|^{2} \lambda(d u) \leq L_{m}|x-y|^{2}$ where $H(x, u)=\gamma(u)$ $x\left(t^{-}\right)$with $|x| \vee|y| \leq m$.

(A3) There exists a positive constant $c$ such that $\mid \ln (1+$ $\gamma(u)) \mid \leq c$ for $\gamma(u)>-1$.

For the simplicity, we define the following notations:

$$
\begin{gathered}
\langle x(t)\rangle=\frac{1}{t} \int_{0}^{t} x(s) d s, \\
x_{*}=\liminf _{t \rightarrow+\infty} x(t), \quad x^{*}=\limsup _{t \rightarrow+\infty} x(t), \\
g(t)=r(t)-\int_{\mathbb{Y}}[\gamma(u)-\ln (1+\gamma(u))] \lambda(d u) .
\end{gathered}
$$

The rest of the paper is arranged as follows. In Section 2, we show that model (3) has a unique positive global solution. Afterward, sufficient and necessary conditions for extinction and stochastic permanence are established in Section 3. Section 4 mainly concentrates on introducing some figures to illustrate the main results. Finally, we close the paper with conclusions and remarks in Section 5.

\section{Global Positive Solution}

The classical existence and uniqueness result for solutions of a stochastic differential delay equation with jumps requires the coefficient functions to satisfy a local Lipschitz condition and a linear growth condition (see, e.g., [17-21]). Clearly, the coefficients of (3) satisfy the local Lipschitz condition, while they fail the linear growth condition. In this section, using the Lyapunov analysis method (mentioned in [12]), we will show that the jump processes can suppress the explosion and the solution of model (3) is positive and global. For later applications, let us cite the following lemma.
Lemma 1. The following inequalities hold:

$$
\begin{gathered}
\ln (x+1) \leq x, \quad x>-1, \\
x^{q} \leq 1+q(x-1), \quad x \geq 0,0 \leq q \leq 1 .
\end{gathered}
$$

Theorem 2. Let assumptions (A1)-(A3) hold. For any given initial value $\xi(t) \in C_{\mathscr{F}_{0}^{b}}\left([-\tau, 0] ; R_{+}\right)$, (3) has a unique positive solution $x(t) \in R_{+}$for any $t \geq 0$ almost surely.

Proof. Since the coefficients of the equation are locally Lipschitz continuous, for any given initial value $\xi(t) \epsilon$ $C_{\mathscr{F}_{0}^{b}}\left([-\tau, 0] ; R_{+}\right)$, there is a unique local solution $x$ on $t \in$ $\left[-\tau, \tau_{e}\right)$, where $\tau_{e}$ is the explosion time. To show that this solution is global, we need to show that $\tau_{e}=+\infty$ a.s. Let $k_{0}>0$ be sufficiently large for $\left(1 / k_{0}\right)<\min _{-\tau \leq t \leq 0} \xi(t) \leq$ $\max _{-\tau \leq t \leq 0} \xi(t)<k_{0}$. For each time integer $k \geq k_{0}$, define the stopping time:

$$
\tau_{k}=\inf \left\{t \in\left[0, \tau_{e}\right): x(t) \leq \frac{1}{k} \text { or } x(t) \geq k\right\}
$$

where throughout this paper we set inf $\emptyset=+\infty$ (as usually $\emptyset$ denotes the empty set). Clearly, $\tau_{k}$ is increasing as $k \rightarrow+\infty$. Set $\tau_{+\infty}=\lim _{k \rightarrow+\infty} \tau_{k}$; hence $\tau_{+\infty} \leq \tau_{e}$ a.s. If we can show that $\tau_{+\infty}=+\infty$ a.s., then $\tau_{e}=+\infty$ a.s. and $x(t) \in R_{+}$a.s. for all $t \geq 0$. In other words, to complete the proof all we need to show is that $\tau_{+\infty}=+\infty$ a.s. To show this statement, let us define a $C^{2}$-function $V: R_{+} \rightarrow R_{+}$by $V(x)=\sqrt{x}-1-0.5 \ln x$. Let $k \geq k_{0}$ and $T>0$ be arbitrary. For $0 \leq t \leq \tau_{k} \wedge T$, applying the Itô's formula, we obtain

$$
\begin{aligned}
& d\left[\int_{t-\tau}^{t} x^{2}(s) d s+V(x(t))\right] \\
& =\left(x^{2}(t)-x^{2}(t-\tau)\right) d t+0.5\left(x^{-0.5}(t)-x^{-1}(t)\right) \\
& \quad \times[x(t)(r(t)-a(t) x(t)+b(t) x(t-\tau)) d t \\
& \left.\quad+\sigma(t) x^{1+\theta}(t) d w(t)\right] \\
& \quad+0.5\left[-0.25 x^{-1.5}(t)+0.5 x^{-2}(t)\right] \sigma^{2}(t) x^{2+2 \theta}(t) d t \\
& \quad+\int_{\mathbb{Y}}\left[(1+\gamma(u))^{0.5}-1-0.5 \gamma(u)\right] \lambda(d u) x^{0.5}(t) d t \\
& \quad+0.5 \int_{\mathbb{Y}}[\gamma(u)-\ln (1+\gamma(u))] \lambda(d u) d t \\
& \quad+\int_{\mathbb{Y}}\left[(1+\gamma(u))^{0.5}-1\right] \widetilde{N}(d t, d u) x^{0.5}(t) \\
& \quad-0.5 \int_{\mathbb{Y}}[\ln (1+\gamma(u))] \widetilde{N}(d t, d u) \\
& \leq\left[x^{2}(t)-x^{2}(t-\tau)\right] d t
\end{aligned}
$$




$$
\begin{aligned}
& +\left[0.5 r(t)\left(x^{0.5}(t)-1\right)\right. \\
& -0.5 a(t) x(t)\left(x^{0.5}(t)-1\right)+x^{2}(t-\tau) \\
& \left.+0.0625 b^{2}(t)\left(x^{0.5}(t)-1\right)^{2}\right] d t \\
& +0.5\left[-0.25 x^{-1.5}(t)+0.5 x^{-2}(t)\right] \sigma^{2}(t) x^{2+2 \theta}(t) d t \\
& +\int_{\mathbb{Y}}\left[(1+\gamma(u))^{0.5}-1-0.5 \gamma(u)\right] \lambda(d u) x^{0.5} d t \\
& +0.5 \int_{\mathbb{Y}}[\gamma(u)-\ln (1+\gamma(u))] \lambda(d u) d t \\
& +0.5 x^{\theta}(t)\left(x^{0.5}(t)-1\right) \sigma(t) d \omega(t) \\
& +\int_{\mathbb{Y}}\left[(1+\gamma(u))^{0.5}-1\right] \widetilde{N}(d t, d u) x^{0.5}(t) \\
& -0.5 \int_{\mathbb{V}} \ln (1+\gamma(u)) \widetilde{N}(d t, d u) \\
& =\left(-0.5 r(t)+0.0625 b^{2}(t)-0.125 b^{2}(t) x^{0.5}(t)\right. \\
& +0.5 r(t) x^{0.5}(t)+0.0625 b^{2}(t) x(t) \\
& +0.5 a(t) x(t)-0.5 a(t) x^{1.5}(t)+x^{2}(t) \\
& \left.+0.25 \sigma^{2}(t) x^{2 \theta}(t)-0.125 \sigma^{2}(t) x^{0.5+2 \theta}(t)\right) d t \\
& +\int_{\mathbb{Y}}\left[(1+\gamma(u))^{0.5}-1-0.5 \gamma(u)\right] \lambda(d u) x^{0.5} d t \\
& +0.5 \int_{\mathbb{Y}}[\gamma(u)-\ln (1+\gamma(u))] \lambda(d u) d t \\
& +0.5 x^{\theta}(t)\left(x^{0.5}(t)-1\right) \sigma(t) d \omega(t) \\
& +\int_{\mathbb{Y}}\left[(1+\gamma(u))^{0.5}-1\right] \widetilde{N}(d t, d u) x^{0.5}(t) \\
& -0.5 \int_{\mathbb{Y}} \ln (1+\gamma(u)) \widetilde{N}(d t, d u) \\
& =F(x(t)) d t+0.5 x^{\theta}(t)\left(x^{0.5}(t)-1\right) \sigma(t) d \omega(t) \\
& +\int_{\mathbb{Y}}\left[(1+\gamma(u))^{0.5}-1\right] \widetilde{N}(d t, d u) x^{0.5}(t) \\
& -0.5 \int_{\mathbb{Y}}[\ln (1+\gamma(u))] \widetilde{N}(d t, d u),
\end{aligned}
$$

where

$$
\begin{aligned}
F(x)= & -0.5 r(t)+0.0625 b^{2}(t)-0.125 b^{2}(t) x^{0.5} \\
& +0.5 r(t) x^{0.5}+0.0625 b^{2}(t) x \\
& +0.5 a(t) x-0.5 a(t) x^{1.5}+x^{2} \\
& +0.25 \sigma^{2}(t) x^{2 \theta}-0.125 \sigma^{2}(t) x^{0.5+2 \theta}
\end{aligned}
$$

$$
\begin{aligned}
& +\int_{\mathbb{V}}\left[(1+\gamma(u))^{0.5}-1-0.5 \gamma(u)\right] \lambda(d u) x^{0.5} \\
& +0.5 \int_{\mathbb{V}}[\gamma(u)-\ln (1+\gamma(u))] \lambda(d u) .
\end{aligned}
$$

From the inequality $x^{q} \leq 1+q(x-1)$ for $x \geq 0,0 \leq q \leq 1$, and assumptions (Al) and (A3), it is easy to see that $F(x)$ is bounded, say by $K$, in $R_{+}$. We therefore obtain that

$$
\begin{aligned}
& d\left[\int_{t-\tau}^{t} x^{2}(s) d s+V(x(t))\right] \\
& \leq K d t+0.5 x^{\theta}(t)\left(x^{0.5}(t)-1\right) \sigma(t) d \omega(t) \\
& \quad+\int_{\mathbb{V}}\left[(1+\gamma(u))^{0.5}-1\right] \widetilde{N}(d t, d u) x^{0.5} \\
& \quad-0.5 \int_{\mathbb{V}}[\ln (1+\gamma(u))] \widetilde{N}(d t, d u) .
\end{aligned}
$$

Integrating both sides from 0 to $t$, and then taking expectations, yields

$$
\begin{aligned}
& E\left[\int_{t-\tau}^{t} x^{2}(s) d s+V(x(t))\right] \\
& \quad \leq \int_{-\tau}^{0} \xi^{2}(s) d s+V(x(0))+K t .
\end{aligned}
$$

Letting $t=\tau_{k} \wedge T$, we obtain that $E V\left(x\left(\tau_{k} \wedge T\right)\right) \leq \int_{-\tau}^{0} \xi^{2}(s) d s+$ $V(x(0))+K T$. Note that for every $\omega \in\left\{\tau_{k} \leq T\right\}, x\left(\tau_{k}, \omega\right)$ equals either $k$ or $1 / k$, and hence $V\left(x\left(\tau_{k}, \omega\right)\right)$ is not less than either $\sqrt{k}-1-0.5 \log (k)$ or $\sqrt{1 / k}-1-0.5 \log (1 / k)=\sqrt{1 / k}-$ $1+0.5 \log (k)$. Consequently,

$$
\begin{aligned}
V\left(x\left(\tau_{k}, \omega\right)\right) \geq & {[\sqrt{k}-1-0.5 \log (k)] } \\
& \wedge\left[\sqrt{\frac{1}{k}}-1+0.5 \log (k)\right] .
\end{aligned}
$$

It then follows from (11) that

$$
\begin{aligned}
\int_{-\tau}^{0} \xi^{2}(s) d s+V(x(0))+K T \\
\geq E\left[1_{\left\{\tau_{k} \leq T\right\}} V\left(x\left(\tau_{k}, \omega\right)\right)\right] \\
\geq P\left\{\tau_{k} \leq T\right\}([\sqrt{k}-1-0.5 \log (k)] \\
\left.\wedge\left[\sqrt{\frac{1}{k}}-1+0.5 \log (k)\right]\right),
\end{aligned}
$$

where $1_{\left\{\tau_{k} \leq T\right\}}$ is the indicator function of $\left\{\tau_{k}\right\}$. Letting $k \rightarrow$ $+\infty$ gives $\lim _{k \rightarrow+\infty} P\left\{\tau_{k} \leq T\right\}=0$. Since $T>0$ is arbitrary, we have $P\left\{\tau_{+\infty}<+\infty\right\}=0$, and so $P\left\{\tau_{+\infty}=+\infty\right\}=1$ as required.

\section{Permanence and Extinction for Model (3)}

Theorem 3. Let assumptions (A1)-(A3) hold. If $g^{*}<0$ and $\inf _{t \in \bar{R}_{+}}[a(t)-b(t+\tau)] \geq 0$; then the population $x(t)$ represented by (3) goes to extinction a.s. 
Proof. Now applying Itô's formula to (3) leads to

$$
\begin{aligned}
& d \int_{t-\tau}^{t} b(s+\tau) x(s) d s+d \ln x(t) \\
& =(b(t+\tau) x(t)-b(t) x(t-\tau)) d t \\
& \quad+\left[r(t)-a(t) x(t)+b(t) x(t-\tau)-\frac{\sigma^{2}(t) x^{2 \theta}(t)}{2}\right. \\
& \left.\quad+\int_{\mathbb{Y}}[\ln (1+\gamma(u))-\gamma(u)] \lambda(d u)\right] d t \\
& +\sigma(t) x^{\theta}(t) d \omega(t)+\int_{\mathbb{Y}} \ln (1+\gamma(u)) \widetilde{N}(d t, d u) .
\end{aligned}
$$

Then we have

$$
\begin{aligned}
& \int_{t-\tau}^{t} b(s+\tau) x(s) d s \\
& \quad-\int_{-\tau}^{0} b(s+\tau) x(s) d s+\ln x(t)-\ln x(0) \\
& =\int_{0}^{t}\left[r(s)-(a(s)-b(s+\tau)) x(s)-\frac{\sigma^{2}(s) x^{2 \theta}(s)}{2}\right] d s \\
& \quad-t \int_{\mathbb{Y}}[\gamma(u)-\ln (1+\gamma(u))] \lambda(d u)+M_{1}(t) \\
& \quad+\int_{0}^{t} \int_{\mathbb{Y}} \ln (1+\gamma(u)) \widetilde{N}(d s, d u),
\end{aligned}
$$

where $M_{1}(t)=\int_{0}^{t} \sigma(s) x^{\theta}(s) d \omega(s)$. The quadratic variation of $M_{1}(t)$ is $\left\langle M_{1}(t), M_{1}(t)\right\rangle=\int_{0}^{t} \sigma^{2}(s) x^{2 \theta}(s) d s$. By virtue of the exponential martingale inequality, for any positive constants $T_{0}, \alpha$, and $\beta$, we have

$$
\mathscr{P}\left\{\sup _{0 \leq t \leq T_{0}}\left[M_{1}(t)-\frac{\alpha}{2}\left\langle M_{1}(t), M_{1}(t)\right\rangle\right]>\beta\right\} \leq e^{-\alpha \beta} .
$$

Choose $T_{0}=k, \alpha=1$, and $\beta=2 \ln k$. Then it follows that

$$
\mathscr{P}\left\{\sup _{0 \leq t \leq k}\left[M_{1}(t)-\frac{1}{2}\left\langle M_{1}(t), M_{1}(t)\right\rangle\right]>2 \ln k\right\} \leq \frac{1}{k^{2}} .
$$

Making use of the Borel-Cantelli lemma yields that, for almost all $\omega \in \Omega$, there is a random integer $k_{0}=k_{0}(\omega)$ such that, for $k \geq k_{0}$,

$$
\sup _{0 \leq t \leq k}\left[M_{1}(t)-\frac{1}{2}\left\langle M_{1}(t), M_{1}(t)\right\rangle\right] \leq 2 \ln k
$$

That is to say, $M_{1}(t) \leq 2 \ln k+(1 / 2)\left\langle M_{1}(t), M_{1}(t)\right\rangle=2 \ln k+$ $(1 / 2) \int_{0}^{t} \sigma^{2}(s) x^{2 \theta}(s) d s$, for all $0 \leq t \leq k, k \geq k_{0}$ a.s. Substituting this inequality into (15), we can obtain that

$$
\begin{aligned}
& \ln x(t)-\ln x(0) \\
& \leq \int_{-\tau}^{0} b(s+\tau) x(s) d s \\
& \quad+\int_{0}^{t}[r(s)-(a(s)-b(s+\tau)) x(s)] d s \\
& \quad-t \int_{\mathbb{Y}}[\gamma(u)-\ln (1+\gamma(u))] \lambda(d u) \\
& \quad+M_{1}(t)+2 \ln k+\int_{0}^{t} \int_{\mathbb{Y}} \ln (1+\gamma(u)) \widetilde{N}(d s, d u),
\end{aligned}
$$

for all $0 \leq t \leq k, k \geq k_{0}$ a.s. In other words, we have shown that for $k-1 \leq t \leq k, k \geq k_{0}$ a.s.,

$$
\begin{aligned}
& t^{-1}\{\ln x(t)-\ln x(0)\} \\
& \leq t^{-1} \int_{-\tau}^{0} b(s+\tau) x(s) d s \\
& \quad+t^{-1} \int_{0}^{t}[r(s)-(a(s)-b(s+\tau)) x(s)] d s \\
& \quad-\int_{\mathbb{Y}}[\gamma(u)-\ln (1+\gamma(u))] \lambda(d u) \\
& +2(k-1)^{-1} \ln k+t^{-1} M_{1}(t) \\
& +t^{-1} \int_{0}^{t} \int_{\mathbb{Y}} \ln (1+\gamma(u)) \widetilde{N}(d s, d u) .
\end{aligned}
$$

Define, for $t \geq 0, M_{2}(t)=\int_{0}^{t} \int_{\mathbb{Y}} \ln (1+\gamma(u)) \widetilde{N}(d s, d u)$. Under assumption (A3), $\left\langle M_{2}\right\rangle(t)=\int_{0}^{t} \int_{\mathbb{Y}} \ln (1+\gamma(u))^{2} \lambda(d u) d s \leq$ $c^{2} t \lambda(\mathbb{Y})$, by the strong law of large numbers for local martingales (see, e.g., [22]); we then obtain

$$
\lim _{t \rightarrow+\infty} \frac{1}{t} \int_{0}^{t} \int_{\mathbb{V}} \ln (1+\gamma(u)) \widetilde{N}(d s, d u)=0 \quad \text { a.s. }
$$

Taking superior limit on both sides of (20) and then making use of (21) yield $\lim \sup _{t \rightarrow+\infty} t^{-1} \ln x(t) \leq g^{*}$. That is to say, if $g^{*}<0$, one can see that $\lim _{t \rightarrow+\infty} x(t)=0$ a.s.

Definition 4 (see, e.g., Bao et al. [11]). Population size $x(t)$ is said to be stochastic permanence if, for arbitrary $\varepsilon>$ 0 , there are constants $\beta>0$ and $M>0$ such that $\liminf _{t \rightarrow+\infty} \mathscr{P}\{x(t) \geq \beta\} \geq 1-\varepsilon{\text { and } \liminf _{t \rightarrow+\infty}} \mathscr{P}\{x(t) \leq$ $M\} \geq 1-\varepsilon$.

Theorem 5. Let assumptions (A1)-(A3) hold. If $g_{*}>0$ and $\theta \leq 1$, then the population $x(t)$ modeled by (3) will be stochastic permanence. 
Proof. First, we prove that for arbitrary $\varepsilon>0$, there is constant $M>0$ such that $\liminf _{t \rightarrow+\infty} \mathscr{P}\{x(t) \leq M\} \geq 1-\varepsilon$.

Let $0.5<p<1$; we compute

$$
\begin{aligned}
& d x^{p}(t)=p x^{p-1}(t) d x(t)+\frac{1}{2} p(p-1) x^{p-2}(t)(d x(t))^{2} \\
& +\int_{\mathbb{Y}}\left[(1+\gamma(u))^{p}-1-p \gamma(u)\right] \lambda(d u) x^{p}(t) d t \\
& +\int_{\mathbb{Y}}\left[(1+\gamma(u))^{p}-1\right] \widetilde{N}(d t, d u) x^{p}(t) \\
& =p x^{p-1}(t) \\
& \times[x(t)(r(t)-a(t) x(t)+b(t) x(t-\tau)) d t \\
& \left.+\sigma(t) x^{1+\theta}(t) d \omega(t)\right] \\
& +\frac{1}{2} p(p-1) \sigma^{2}(t) x^{p+2 \theta}(t) d t \\
& +\int_{\mathbb{Y}}\left[(1+\gamma(u))^{p}-1-p \gamma(u)\right] \lambda(d u) x^{p}(t) d t \\
& +\int_{\mathbb{Y}}\left[(1+\gamma(u))^{p}-1\right] \widetilde{N}(d t, d u) x^{p}(t) \\
& \leq\left[r(t) p x^{p}(t)+\frac{p^{2} b^{2}(t) x^{2 p}(t)}{4}+x^{2}(t-\tau)\right] d t \\
& +p \sigma(t) x^{p+\theta}(t) d \omega(t) \\
& -\frac{1}{2} p(1-p) \sigma^{2}(t) x^{p+2 \theta}(t) d t \\
& +\int_{\mathbb{Y}}\left[(1+\gamma(u))^{p}-1-p \gamma(u)\right] \lambda(d u) x^{p}(t) d t \\
& +\int_{\mathbb{Y}}\left[(1+\gamma(u))^{p}-1\right] \widetilde{N}(d t, d u) x^{p}(t) \\
& =F(x(t)) d t \\
& -\left[\varepsilon_{1} x^{p}(t)+e^{\varepsilon_{1} \tau} x^{2}(t)-x^{2}(t-\tau)\right] d t \\
& +p \sigma(t) x^{p+2 \theta}(t) d \omega(t) \\
& +\int_{\mathbb{Y}}\left[(1+\gamma(u))^{p}-1\right] \widetilde{N}(d t, d u) x^{p}(t),
\end{aligned}
$$

where $\varepsilon_{1}$ is a positive constant and

$$
\begin{aligned}
F(x)= & e^{\varepsilon_{1} \tau} x^{2}+\left(\varepsilon_{1}+r(t) p\right) x^{p} \\
& +\frac{p^{2} b^{2}(t) x^{2 p}}{4}-\frac{1}{2} p(1-p) \sigma^{2}(t) x^{p+2 \theta} \\
& +\int_{\mathbb{Y}}\left[(1+\gamma(u))^{p}-1-p \gamma(u)\right] \lambda(d u) x^{p} .
\end{aligned}
$$

Making use of (6) in Lemma 1 , we obtain $\int_{\mathbb{Y}}\left[(1+\gamma(u))^{p}-1-\right.$ $p \gamma(u)] \lambda(d u) x^{p} \leq 0$. In view of the inequality above, $\theta>0.75$ and $0.5<p<1$, we have that $F(x)$ is bounded in $\bar{R}_{+}$; namely, $M_{3}=\sup _{x \in \bar{R}_{+}} F(x)<+\infty$. Therefore

$$
\begin{aligned}
d x^{p}(t) \leq[ & \left.M_{3}-\varepsilon_{1} x^{p}(t)-e^{\varepsilon_{1} \tau} x^{2}(t)+x^{2}(t-\tau)\right] d t \\
& +p \sigma(t) x^{p+2 \theta}(t) d \omega(t) \\
& +\int_{\mathbb{Y}}\left[(1+\gamma(u))^{p}-1\right] \widetilde{N}(d t, d u) x^{p}(t) .
\end{aligned}
$$

Once again by the Itô's formula we have

$$
\begin{aligned}
d\left[e^{\varepsilon_{1} t} x^{p}(t)\right] \\
=e^{\varepsilon_{1} t}\left[\varepsilon_{1} x^{p}(t) d t+d x^{p}(t)\right] \\
\leq e^{\varepsilon_{1} t}\left[M_{3}-e^{\varepsilon_{1} \tau} x^{2}(t)+x^{2}(t-\tau)\right] \\
\quad+e^{\varepsilon_{1} t} p \sigma(t) x^{p+2 \theta}(t) d \omega(t) \\
\quad+e^{\varepsilon_{1} t}\left(\int_{\mathbb{Y}}\left[(1+\gamma(u))^{p}-1\right] \widetilde{N}(d t, d u) x^{p}(t)\right) .
\end{aligned}
$$

We hence derive that

$$
\begin{aligned}
e^{\varepsilon_{1} t} E[ & \left.x^{p}(t)\right] \\
\leq & x^{p}(0)+\frac{e^{\varepsilon_{1} t} M_{3}}{\varepsilon_{1}}-\frac{M_{3}}{\varepsilon_{1}}-E \int_{0}^{t} e^{\varepsilon_{1} s+\varepsilon_{1} \tau} x^{2}(s) d s \\
& +E \int_{0}^{t} e^{\varepsilon_{1} s} x^{2}(s-\tau) d s \\
= & x^{p}(0)+\frac{e^{\varepsilon_{1} t} M_{3}}{\varepsilon_{1}}-\frac{M_{3}}{\varepsilon_{1}} \\
& -E \int_{0}^{t} e^{\varepsilon_{1} s+\varepsilon_{1} \tau} x^{2}(s) d s+E \int_{-\tau}^{t-\tau} e^{\varepsilon_{1} s+\varepsilon_{1} \tau} x^{2}(s) d s \\
\leq & x^{p}(0)+\frac{e^{\varepsilon_{1} t} M_{3}}{\varepsilon_{1}}-\frac{M_{3}}{\varepsilon_{1}}+\int_{-\tau}^{0} e^{\varepsilon_{1} s+\varepsilon_{1} \tau} x^{2}(s) d s .
\end{aligned}
$$

This implies immediately that $\limsup _{t \rightarrow+\infty} E\left[x^{p}(t)\right] \leq$ $M_{3} / \varepsilon_{1}$. Now, for any $\varepsilon>0$ and $M=\left(M_{3} / \varepsilon_{1}\right)^{1 / p} / \varepsilon^{1 / p}$, then by Chebyshev's inequality,

$$
\mathscr{P}\{x(t)>M\}=\mathscr{P}\left\{x^{p}(t)>M^{p}\right\} \leq \frac{E\left[x^{p}(t)\right]}{M^{p}} .
$$

Hence $\lim \sup _{t \rightarrow+\infty} \mathscr{P}\{x(t)>M\} \leq \varepsilon$. This implies $\liminf \operatorname{int+\infty }_{t \rightarrow} \mathscr{P}\{x(t) \leq M\} \geq 1-\varepsilon$.

Next, we claim that for arbitrary $\varepsilon>0$, there is constant $\beta>0$ such that $\liminf _{t \rightarrow+\infty} \mathscr{P}\{x(t) \geq \beta\} \geq 1-\varepsilon$.

Obviously,

$$
\begin{gathered}
\lim _{\kappa \rightarrow 0^{+}} \int_{\mathbb{Y}}\left[\frac{1}{\kappa(1+\gamma(u))^{\kappa}}-\frac{1}{\kappa}\right] \lambda(d u) \\
\quad=\int_{\mathbb{Y}} \ln \left(\frac{1}{1+\gamma(u)}\right) \lambda(d u) \\
=-\int_{\mathbb{V}} \ln (1+\gamma(u)) \lambda(d u) .
\end{gathered}
$$


Hence if $g_{*}>0$, we can find a sufficiently small $\kappa>0$ such that

$$
\begin{aligned}
r(t) & -\int_{\mathbb{V}} \gamma(u) \lambda(d u) \\
& -\int_{\mathbb{V}}\left[\frac{1}{\kappa(1+\gamma(u))^{\kappa}}-\frac{1}{\kappa}\right] \lambda(d u)>0 .
\end{aligned}
$$

Define $y=1 / x$ for $x>0$. Then, by Itô's formula (see, e.g., [23, Theorem 2.5]),

$$
\begin{aligned}
d y(t)= & y(t)[- \\
& \quad+\int_{\mathbb{Y}}\left(\frac{1}{1+\gamma(u)}-1+\gamma(t) x(t-\tau)+\sigma^{2}(t) y^{-2 \theta}\right. \\
& +a(t) d t-\sigma(t) y^{1-\theta} d w(t) \\
& +y(t) \int_{\mathbb{Y}}\left(\frac{1}{1+\gamma(u)}-1\right) \widetilde{N}(d t, d u) .
\end{aligned}
$$

Define $z=y^{\kappa}$, where $\kappa$ satifies (29). In view of Itô's formula,

$$
\begin{aligned}
d z(t)= & \kappa y^{\kappa-2}(t) \\
& \times\left\{-y^{2}(t)\left[r(t)-\int_{\mathbb{V}} \gamma(u) \lambda(d u)\right.\right. \\
& -\int_{\mathbb{V}}\left(\frac{1}{\kappa(1+\gamma(u))^{\kappa}}-\frac{1}{\kappa}\right) \lambda(d u) \\
& +b(t) x(t-\tau)] \\
& +a(t) y(t)+\sigma^{2}(t) y^{2-2 \theta}(t) \\
& \left.+0.5 \sigma^{2}(t)(\kappa-1) y^{2-2 \theta}(t)\right\} d t \\
& -\sigma(t) \kappa y^{\kappa-\theta}(t) d w(t) \\
+ & y^{\kappa}(t) \int_{\mathbb{V}}\left(\frac{1}{(1+\gamma(u))^{\kappa}}-1\right) \widetilde{N}(d t, d u) .
\end{aligned}
$$

Now, let $\eta>0$ be sufficiently small satisfying

$$
\begin{aligned}
r_{*} & -\varepsilon-\int_{\mathbb{Y}} \gamma(u) \lambda(d u) \\
& -\int_{\mathbb{Y}}\left[\frac{1}{\kappa(1+\gamma(u))^{\kappa}}-\frac{1}{\kappa}\right] \lambda(d u)>\frac{\eta}{\kappa} .
\end{aligned}
$$

Define $V=e^{\eta t} z=e^{\eta t} y^{\kappa}$. By virtue of Itô's formula,

$$
\begin{aligned}
& d V(t)=\kappa e^{\eta t} y^{\kappa-2}(t) \\
& \times\left\{-y^{2}(t)\left[r(t)-\int_{\mathbb{Y}} \gamma(u) \lambda(d u)\right.\right. \\
& -\int_{\mathbb{V}}\left(\frac{1}{\kappa(1+\gamma(u))^{\kappa}}-\frac{1}{\kappa}\right) \lambda(d u) \\
& \left.+b(t) x(t-\tau)-\frac{\eta}{\kappa}\right] \\
& +a(t) y(t)+0.5 \sigma^{2}(t) y^{2-2 \theta}(t) \\
& \left.+0.5 \sigma^{2}(t) \kappa y^{2-2 \theta}(t)\right\} d t \\
& -\sigma(t) \kappa e^{\eta t} y^{\kappa-\theta}(t) d w(t) \\
& +e^{\eta t} y^{\kappa}(t) \int_{\mathbb{Y}}\left(\frac{1}{(1+\gamma(u))^{\kappa}}-1\right) \widetilde{N}(d t, d u) \\
& \leq \kappa e^{\eta t} y^{\kappa-2}(t)\left\{-y^{2}(t)\left[r_{*}-\varepsilon-\int_{\mathbb{Y}} \gamma(u) \lambda(d u)\right.\right. \\
& -\int_{\mathbb{Y}}\left(\frac{1}{\kappa(1+\gamma(u))^{\kappa}}-\frac{1}{\kappa}\right) \\
& \times \lambda(d u)] \\
& -\frac{\eta}{\kappa}+a(t) y(t)+\sigma^{2}(t) y^{2-2 \theta}(t) \\
& \left.+0.5 \sigma^{2}(t)(\kappa-1) y^{2-2 \theta}(t)\right\} d t \\
& -\sigma(t) \kappa e^{\eta t} y^{\kappa-\theta}(t) d w(t) \\
& +e^{\eta t} y^{\kappa}(t) \int_{\mathbb{V}}\left(\frac{1}{(1+\gamma(u))^{\kappa}}-1\right) \widetilde{N}(d t, d u) \\
& =e^{\eta t} K(y(t)) d t-\sigma(t) \kappa e^{\eta t} y^{\kappa-\theta}(t) d w(t) \\
& +e^{\eta t} y^{\kappa}(t) \int_{\mathbb{Y}}\left(\frac{1}{(1+\gamma(u))^{\kappa}}-1\right) \widetilde{N}(d t, d u),
\end{aligned}
$$

for $t \geq T$. Note that $K(y)$ is upper bounded in $R_{+}$; namely, $G=\sup _{x \in R_{+}} K(y)<+\infty$. Consequently,

$$
\begin{aligned}
d V(t) \leq & G e^{\eta t} d t-\sigma(t) \kappa e^{\eta t} x^{\theta-\kappa}(t) d w(t) \\
& +e^{\eta t} y^{\kappa}(t) \int_{\mathbb{Y}}\left(\frac{1}{(1+\gamma(u))^{\kappa}}-1\right) \widetilde{N}(d t, d u),
\end{aligned}
$$

for sufficiently large $t$. Integrating both sides of the above inequality and then taking expectations give 


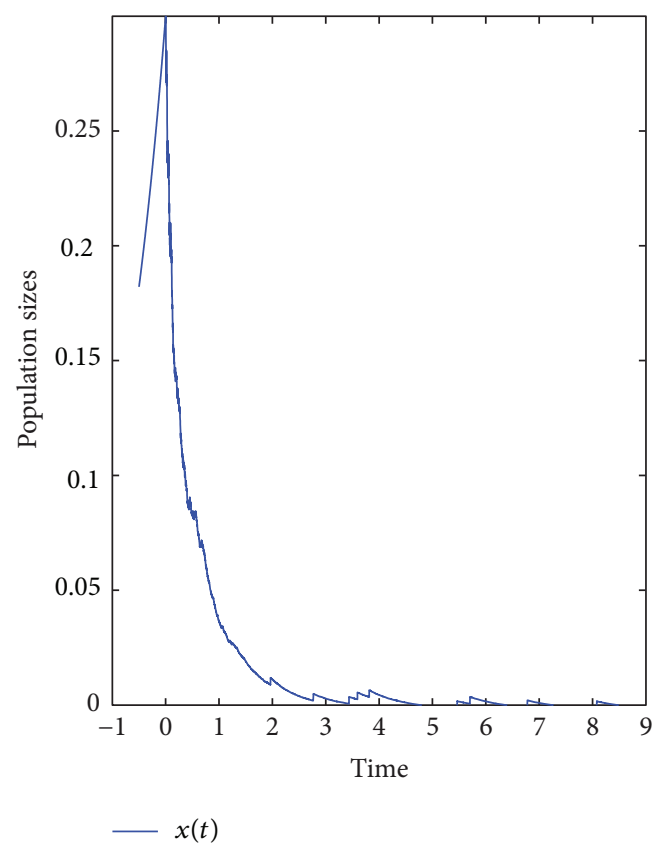

(a)

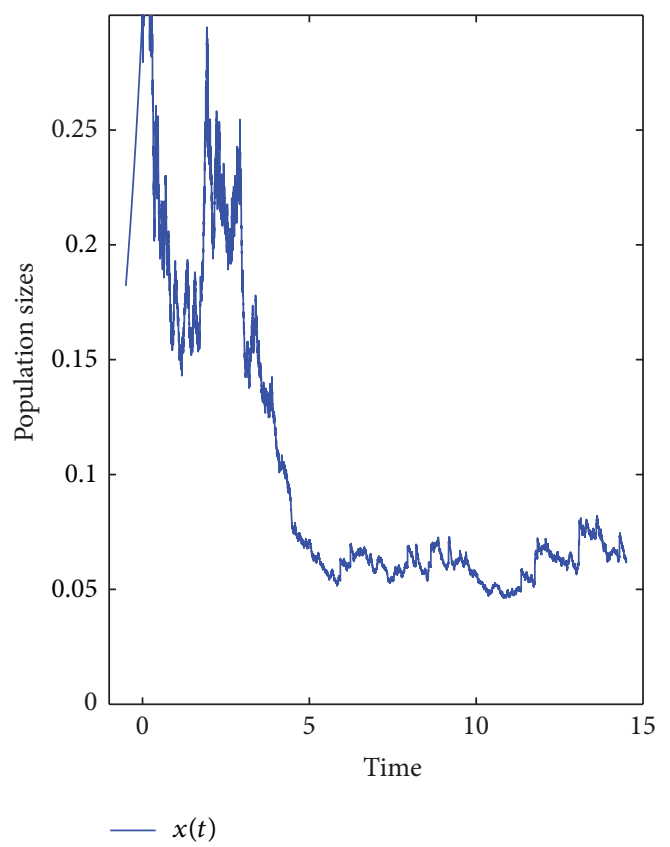

(b)

FIgURE 1: The horizontal axis and the vertical axis in this represent the time $t$ and the population size $x(t)($ step size $\Delta t=0.001)$.

$\limsup _{t \rightarrow+\infty} E\left[x^{-\kappa}(t)\right] \leq G / \eta=M_{4}$. So for any $\varepsilon>0$, set $\beta=\varepsilon^{1 / \kappa} / M_{4}^{1 / \kappa}$. Then the desired assertion follows from the Chebyshev's inequality [24-26]. This completes the whole proof.

Remark 6. Obviously, if assumptions (A1)-(A3) hold, $\theta \leq$ 1 , inf $t \in \bar{R}_{+}[a(t)-b(t+\tau)] \geq 0, \lim _{t \rightarrow+\infty} r(t)$ exists, and $\lim _{t \rightarrow+\infty} r(t) \neq 0$, then Theorems 3 and 5 establish the sufficient and necessary conditions for stochastic permanence and extinction of model (3).

Remark 7. In line with $g^{*}=r^{*}-\int_{\mathbb{Y}}[\gamma(u)-\ln (1+\gamma(u))] \lambda(d u)$ in Theorem 3 and $g_{*}=r_{*}-\int_{\mathbb{Y}}[\gamma(u)-\ln (1+\gamma(u))] \lambda(d u)$ in Theorem 5, where $\int_{\mathbb{Y}}[\gamma(u)-\ln (1+\gamma(u))] \lambda(d u)>0$ (see Lemma 2.2 in [12]), we found that the jump process exists considerable level of detriment to permanence and leads to the extinction of the population, which conforms to biological significance.

Remark 8. If $\theta=1, \tau=0, b(t)=0$, and $\gamma(u)=0$ which means the jump process degenerates to zero, then our result about extinction and permanence coincide with the ones in paper [27]. Moreover, in view of Theorem 5, we found that time delay has no impact on the permanence.

\section{Examples and Numerical Simulations}

In this section, we will use the Euler scheme (see, e.g., [28]) to illustrate the analytical findings.

Here, we choose $r(t)=0.2+0.01 \sin t, a(t)=0.15$, $b(t)=0.1, \sigma^{2}(t)=0.64, \theta=0.8, \mathbb{Y}=(0,+\infty), \lambda(\mathbb{Y})=1$, $\xi(t)=0.3 e^{t-0.5}, t \in[0,0.5]$, and step size $\Delta t=0.001$.
The only difference between conditions of Figures 1(a) and 1 (b) is that the representations of $\gamma(u)$ are different. In Figure $1(\mathrm{a})$, we choose $\gamma(u)=0.7935$, and then $g^{*}=-0.01<$ 0 . In view of Theorem 3 , population $x(t)$ will go to extinction. In Figure $1(\mathrm{~b})$, we consider $\gamma(u)=0.7045$, and then $g_{*}=$ $0.02>0$. Making use of Theorem 5 , the population $x(t)$ will be stochastic permanence. By the numerical simulations, we can find that the jump process can affect the properties of the population model significantly.

\section{Conclusions and Remarks}

In this paper, we investigate the permanence and extinction of a stochastic delay logistic model with jumps. Sufficient and necessary conditions for extinction are established as well as stochastic permanence.

Besides, some interesting topics deserve further consideration. One may propose some more realistic but complex models, such as introducing the colored noise into the model [29]. Another significant problem is devoted to multidimensional stochastic delay model with jumps, and these investigations are in progress.

\section{Conflict of Interests}

The authors declare that there is no conflict of interests regarding the publication of this paper.

\section{Acknowledgments}

This paper is supported by the National Natural Science Foundation of China (11271101), the NNSF of Shandong Province in China (ZR2010AQ021), and the Scientific 
Research Foundation of Harbin Institute of Technology at Weihai (HIT (WH) 201319).

\section{References}

[1] H. I. Freedman and J. Wu, "Periodic solutions of single-species models with periodic delay," SIAM Journal on Mathematical Analysis, vol. 23, pp. 689-701, 1992.

[2] Y. Kuang and H. L. Smith, "Global stability for infinite delay Lotka-Volterra type systems," Journal of Differential Equations, vol. 103, no. 2, pp. 221-246, 1993.

[3] X. He and K. Gopalsamy, "Persistence, attractivity, and delay in facultative mutualism," Journal of Mathematical Analysis and Applications, vol. 215, no. 1, pp. 154-173, 1997.

[4] K. Golpalsamy, Stability and Oscillations in Delay Differential Equations of Population Dynamics, Kluwer Academic, Dordrecht, The Netherlands, 1992.

[5] R. M. May, Stability and Complexity in Model Ecosystems, Princeton University Press, 1973.

[6] F. Wei and K. Wang, "The existence and uniqueness of the solution for stochastic functional differential equations with infinite delay," Journal of Mathematical Analysis and Applications, vol. 331, no. 1, pp. 516-531, 2007.

[7] Q. Meng and H. Jiang, "Robust stochastic stability analysis of Markovian switching genetic regulatory networks with discrete and distributed delays," Neurocomputing, vol. 74, no. 1-3, pp. 362-368, 2010.

[8] X. Li, A. Gray, D. Jiang, and X. Mao, "Sufficient and necessary conditions of stochastic permanence and extinction for stochastic logistic populations under regime switching," Journal of Mathematical Analysis and Applications, vol. 376, no. 1, pp. 1128, 2011.

[9] J. Chunyan, J. Daqing, S. Ningzhong, and D. O’Regan, “Existence, uniqueness, stochastic persistence and global stability of positive solutions of the logistic equation with random perturbation," Mathematical Methods in the Applied Sciences, vol. 30, no. 1, pp. 77-89, 2007.

[10] M. Liu and K. Wang, "Population dynamical behavior of LotkaVolterra cooperative systems with random perturbations," Discrete and Continuous Dynamical Systems, vol. 33, pp. 2495-2522, 2013.

[11] J. Bao, X. Mao, G. Yin, and C. Yuan, "Competitive LotkaVolterra population dynamics with jumps," Nonlinear Analysis: Theory, Methods and Applications, vol. 74, no. 17, pp. 6601-6616, 2011.

[12] J. Bao and C. Yuan, "Stochastic population dynamics driven by Lévy noise," Journal of Mathematical Analysis and Applications, vol. 391, no. 2, pp. 363-375, 2012.

[13] M. Liu and K. Wang, "Dynamics of a Leslie-Gower Holling-type II predatorprey system with Levy jumps," Nonlinear Analysis: Theory, Methods \& Applications, vol. 85, pp. 204-213, 2013.

[14] M. Liu and K. Wang, "Stochastic Lotka-Volterra systems with Levy noise jumps," Journal of Mathematical Analysis and Applications, vol. 410, pp. 750-763, 2014.

[15] D. Applebaum, Levy Processes and Stochastics Calculus, Cambridge University Press, 2nd edition, 2009.

[16] R. Situ, Theory of Stochastic Differential Equations with Jumps and Applications, Springer, 2005.

[17] N. Jacob, Y. Wang, and C. Yuan, "Stochastic differential delay equations with jumps, under nonlinear growth condition," Stochastics, vol. 81, pp. 571-588, 2009.
[18] J. Bao and C. Yuan, "Comparison theorem for stochastic differential delay equations with jumps," Acta Applicandae Mathematicae, vol. 116, no. 2, pp. 119-132, 2011.

[19] C. Yuan and X. Mao, "Stability of stochastic delay hybrid systems with jumps," European Journal of Control, vol. 16, no. 6, pp. 595-608, 2010.

[20] B. Oksendal and A. Sulem, Applied Stochastic Control of Jump Diffusions, Springer, Berlin, Germany, 2005.

[21] N. Ikeda and S. Watanabe, Stochastic Differential Equations and Diffusion Processes, North-Holland Publishing Company, Amsterdam, The Netherlands, 1981.

[22] R. Lipster, "A strong law of large numbers for local martingales," Stochastics, vol. 3, pp. 217-228, 1980.

[23] H. Kunita, "Itô's stochastic calculus: Its surprising power for applications," Stochastic Processes and their Applications, vol. 120, no. 5, pp. 622-652, 2010.

[24] M. Liu and K. Wang, "Analysis of a stochastic autonomous mutualism model," Journal of Mathematical Analysis and Applications, vol. 402, pp. 392-403, 2013.

[25] X. Li and X. Mao, "Population dynamical behavior of nonautonomous Lotka-Volterra competitive system with random perturbation," Discrete and Continuous Dynamical Systems, vol. 24, no. 2, pp. 523-545, 2009.

[26] C. Lu and X. H. Ding, "Persistence and extinction for a stochastic logistic model with infinite delay," Electronic Journal of Differential Equations, vol. 262, pp. 1-16, 2013.

[27] M. Liu and K. Wang, "Persistence and extinction in stochastic non-autonomous logistic systems," Journal of Mathematical Analysis and Applications, vol. 375, no. 2, pp. 443-457, 2011.

[28] P. Protter and D. Talay, "The Euler scheme for Lévy driven stochastic differential equations," The Annals of Probability, vol. 25, no. 1, pp. 393-423, 1997.

[29] X. Mao and C. Yuan, Stochastic Differential Equations with Markovian Switching, Imperial College Press, 2006. 


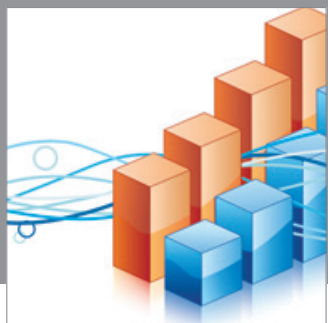

Advances in

Operations Research

mansans

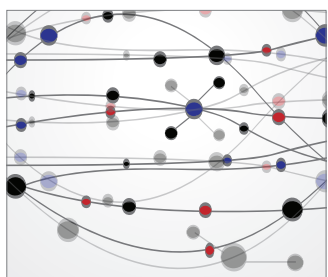

The Scientific World Journal
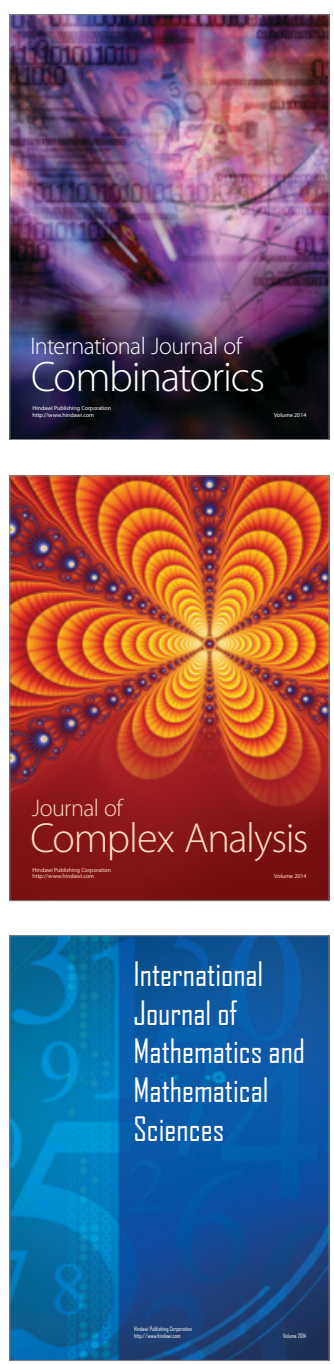
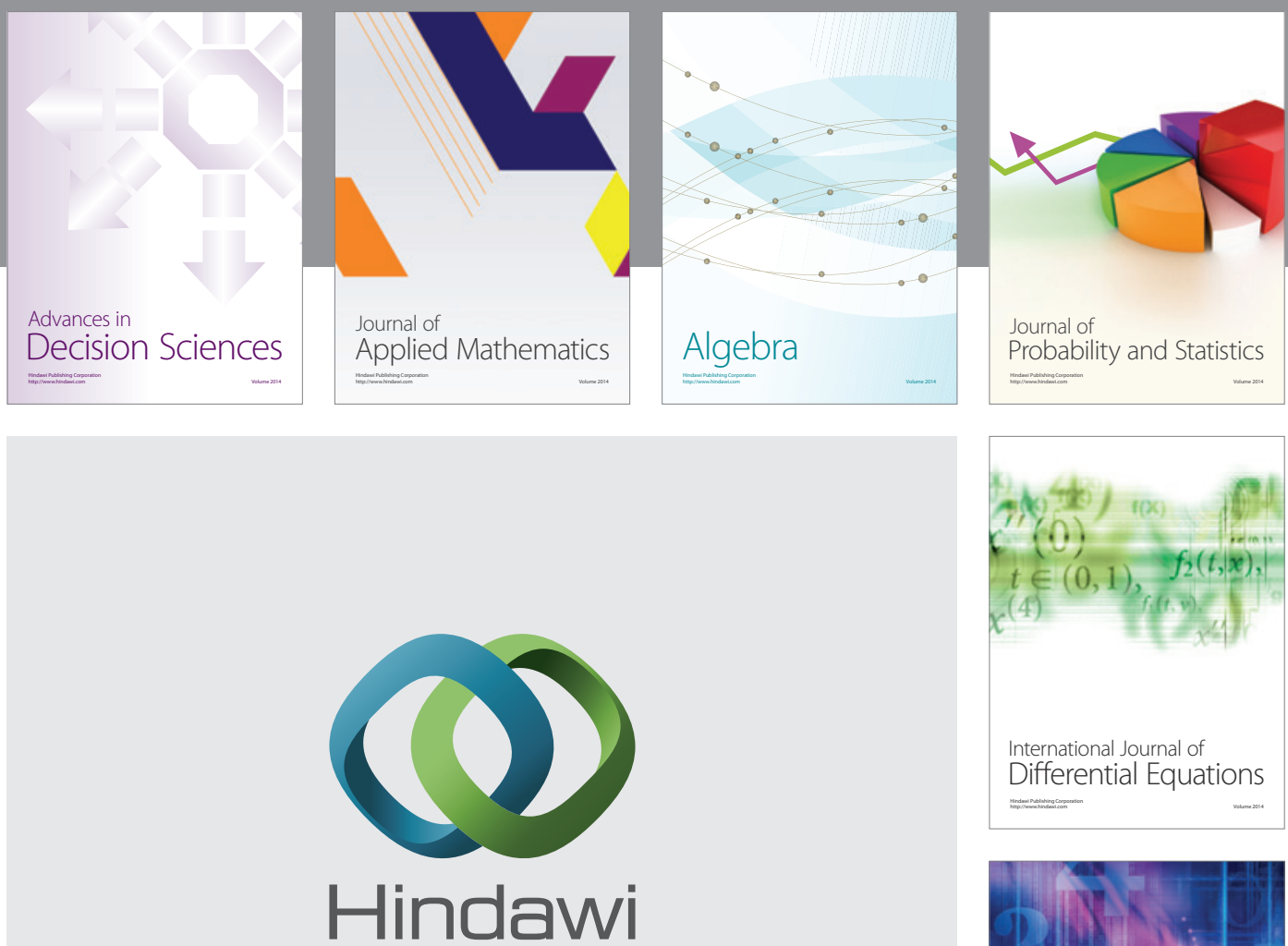

Submit your manuscripts at http://www.hindawi.com
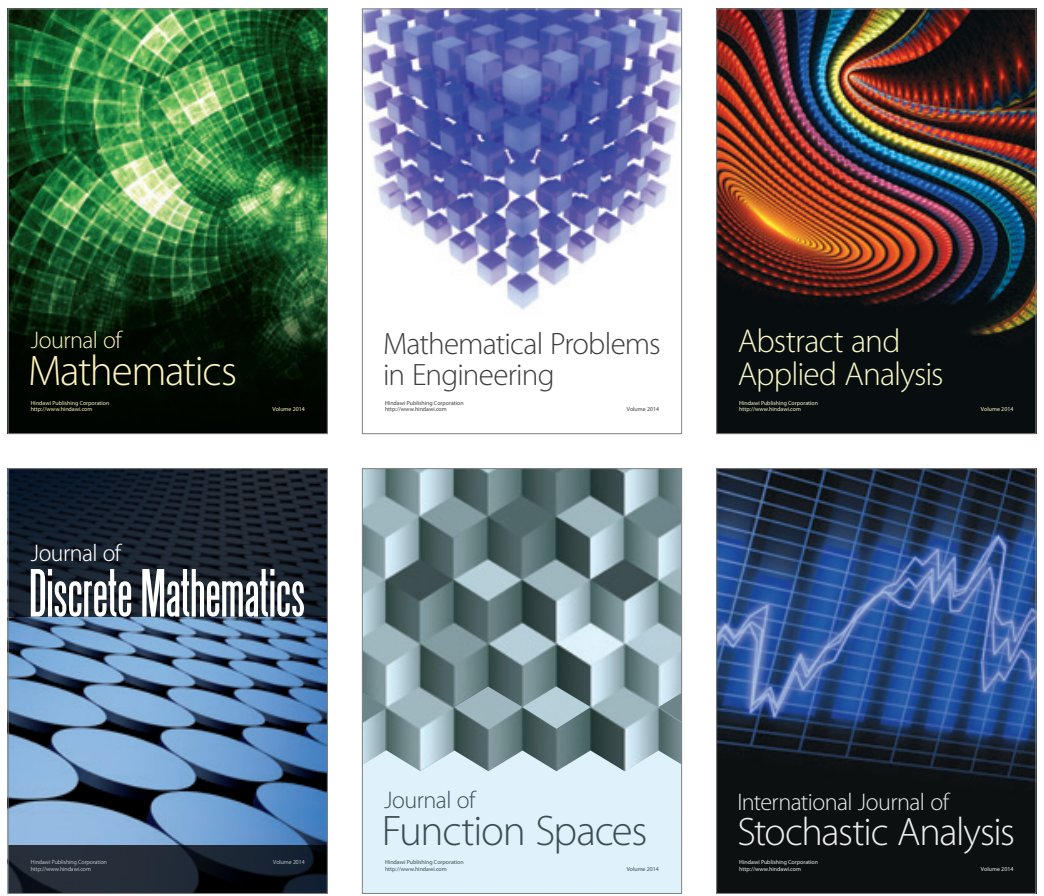

Journal of

Function Spaces

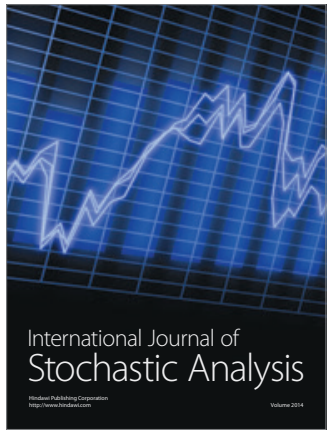

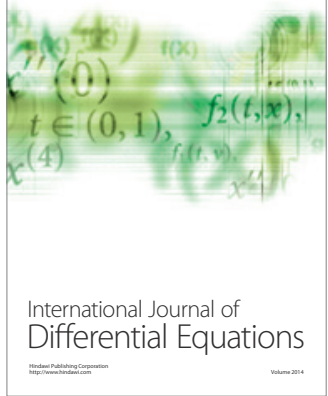
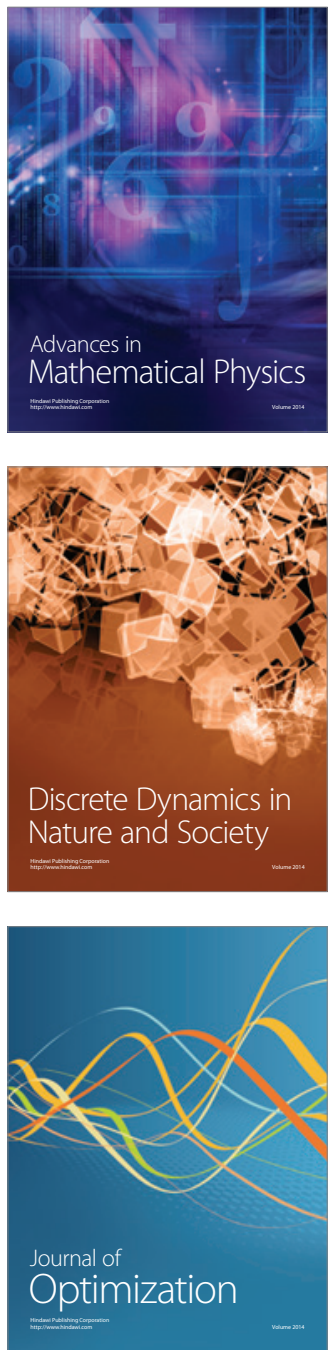